DOI 10.29184/1980-7813.rcfmc.198.vol.13.n2.2018

\title{
ANATOMOFISIOLOGIA DO ESTRESSE E O PROCESSO DE ADOECIMENTO
}

\section{ANATOMOPHYSIOLOGY OF STRESS AND THE PROCESS OF ILLNESS}

Thalita Pereira de Oliveira Rocha ${ }^{1}$ *; Mateus Santos Matos ${ }^{1}$; Flávia Batista Correa ${ }^{1}$; Caio Oliveira Silva ${ }^{1}$; Rogério da Silva Burla $^{2}$

1 - Graduando(a) em Medicina pela Faculdade de Medicina de Campos - Rio de Janeiro

2 - Engenheiro agrônomo pela Universidade Estadual do Norte Fluminense, mestre em engenharia ambiental pelo Instituto Federal Fluminense, graduando em Medicina pela Faculdade de Medicina de Campos - Rio de Janeiro

*Autor de Contato: Thalita Pereira de Oliveira Rocha - (32) 991065270; thalitapereiradeoliveira@gmail.com

\section{RESUMO}

A organização produtiva contemporânea tem imposto aos cidadãos um estilo de vida competitivo, com ameaça de desemprego, longas jornadas de trabalho, necessidade de consumir, além de outras dificuldades. Durante eventos estressantes ocorre uma resposta do eixo hipotálamo-hipófise-adrenal que secreta o hormônio cortisol. Dependendo da duração e intensidade do estressor, o nível elevado de cortisol pode ocasionar patologias. O presente estudo buscou compreender as bases anatômicas e fisiológicas do processo de adoecimento causado pelo estresse crônico. Dentre as patologias mais estudadas causadas pelo hipercortisolismo estão a hipertensão arterial, aumento da glicose sanguínea, fraqueza muscular, estrias, osteoporose, obesidade, degeneração do encéfalo e a redução da imunidade. É importante salientar que as pessoas emitem respostas diferentes ao estresse, de forma que os sinais e sintomas não serão iguais entre os acometidos. Por fim, toda iniciativa pública ou privada que estabeleça a estabilidade social, econômica e emocional pode suprimir as patologias associadas ao estresse.

Palavras-chaves: eixo hipotálamo-hipófise-adrenal, cortisol, patologias.

\begin{abstract}
The contemporary production organization has imposed on citizens a competitive lifestyle, with the threat of unemployment, long working hours, the need to consume, as well as other difficulties. During stressful events, a hypothalamic-pituitary-adrenal axis response that secretes the hormone cortisol occurs. Depending on the duration and intensity of the stressor, the high level of cortisol may cause pathologies. The present study sought to understand the anatomical and physiological bases of the process of illness caused by chronic stress. Among the most studied pathologies caused by hypercortisolism are hypertension, increased blood glucose, muscle weakness, stretch marks, osteoporosis, obesity, degeneration of the brain and reduction of immunity. It is important to emphasize that people emit different responses to stress, so that the signs and symptoms will not be equal among the affected. Finally, any public or private initiative that establishes social, economic and emotional stability can suppress the pathologies associated with stress.

Keywords: hypothalamic-pituitary-adrenal axis, cortisol, pathologies.
\end{abstract}




\section{INTRODUÇÃO}

O estresse ocorre quando um indivíduo recebe um estímulo e o interpreta como algo nocivo ao seu equilíbrio interno. Quando a pessoa entende que ela possui os meios adequados para enfrentar o agente estressor, o estresse é minimizado ou nem sequer é percebido. Por outro lado, se a pessoa julga que não possui as ferramentas necessárias para lidar com o estressor, ela estará sob estresse (QUESADA, 2013). Desta forma, percebe-se o caráter subjetivo do estresse, que dependerá de fatores genéticos, cognitivos e experiências anteriores (LUPIEN et al., 2007).

O estresse, enquanto, função normal no organismo humano atua como promotor da motivação, da busca por melhorias nas atividades cotidianas, estimulando os indivíduos para a superação dos desafios. Este é o eustress, estresse fisiológico do organismo que funciona como elemento impulsionador (SILVA, 2005).

Contudo, a rotina e o estilo de vida contemporâneo tornaram-se extremamente frenéticos quanto à exigência por resultados e produtividade. A presença da competitividade nas relações humanas tem desencadeado emoções negativas quanto à busca por poder, sucesso e dinheiro, levando ao aumento exagerado do estresse, além de ocasionar inúmeras patologias físicas e mentais. Este é o distress, que é anormal e patológico (SILVA, 2005).

Quando há um agente estressor físico ou emocional, atuando sobre o organismo, ocorre a ativação da amígdala (Figura 1), uma estrutura encefálica que faz parte do sistema límbico, uma área cerebral associada, entre outras coisas, com a elaboração das emoções e a tradução destas em sinais bioquímicos (OLIVEIRA, 2013).

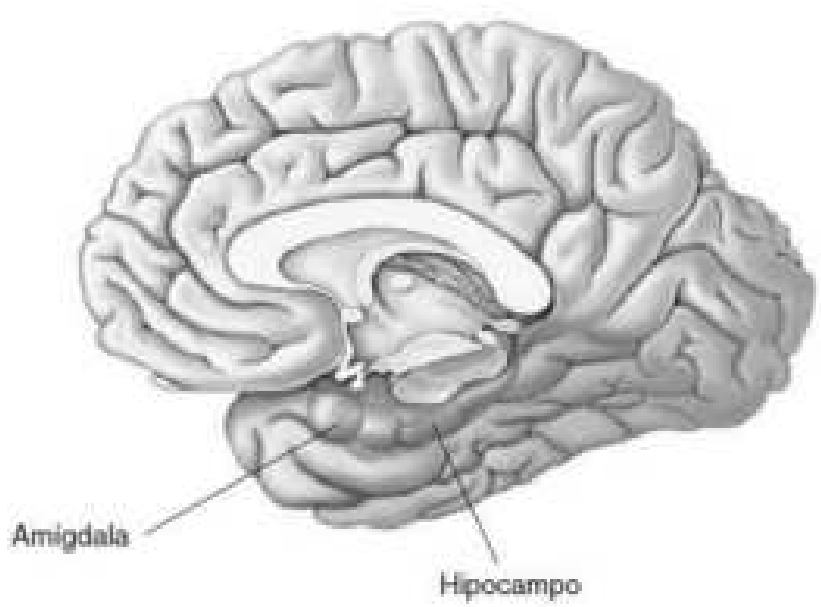

Figura 1 - Destaque da amígdala e hipocampo na vista medial do lobo temporal, após, secção sagital do encéfalo. Adaptado de Bear; Connors; Paradiso (2017).
Na situação de estresse, a resposta neuronal da amígdala estimula a resposta hormonal do hipotálamo induzindo a liberação do fator liberador de corticotropina (CRH), que por sua vez estimula a hipófise ou pituitária a liberar outro hormônio, o adrenocorticotrópico (ACTH), na corrente sanguínea, que irá estimular as glândulas adrenais (zona fasciculada) a liberar o hormônio cortisol que atuará na resposta corporal ao estresse (OLIVEIRA, 2013).

Simultaneamente, o hipotálamo atua diretamente sobre o sistema nervoso autônomo para que ele desencadeie, imediatamente, a reação ao estresse. $\mathrm{O}$ corpo é então preparado para a reação de luta ou fuga através de uma via dupla: uma resposta nervosa de curta duração e uma resposta endócrina (hormonal) (Figura 2), de maior duração (BEAR; CONNORS; PARADISO, 2017; OLIVEIRA, 2013).

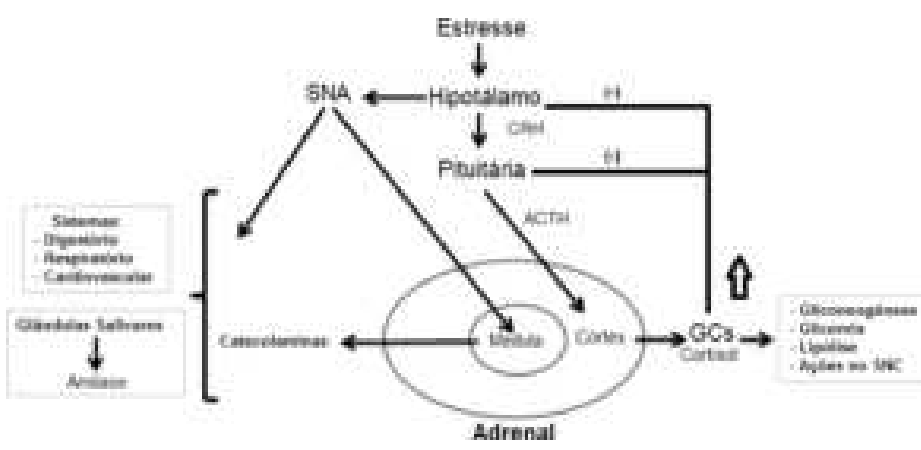

Figura 2 - Estimulação do eixo hipotálamo-hipófise-adrenal e sistema nervoso autônomo em uma situação de estresse. Adaptado de Lipp (2010).

As glândulas adrenais estão situadas imediatamente sobre os rins, sendo por isso, também conhecidas como glândulas suprarrenais (Figura 3).

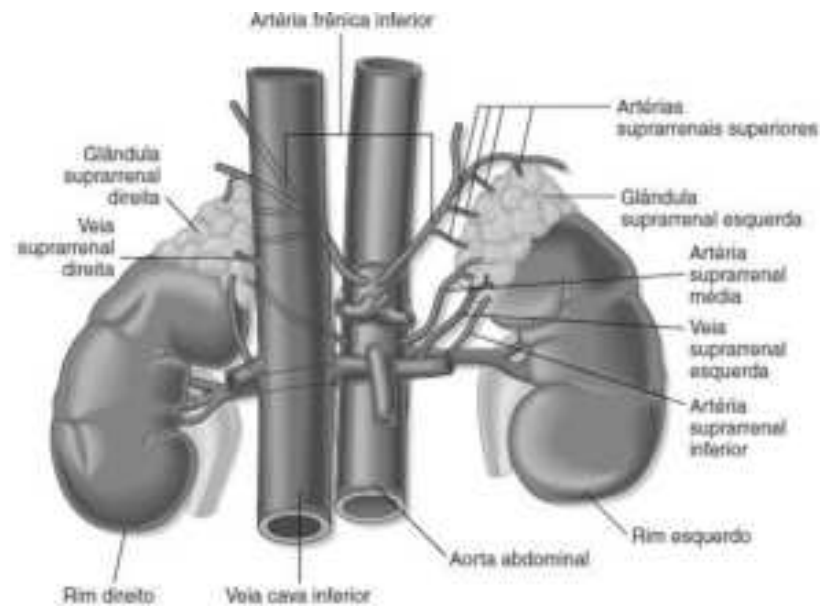

Figura 3 - Relação das glândulas adrenais com os rins. Adaptado de Koeppen; Stanton (2009). 
Embriologicamente, as glândulas adrenais são semelhantes à hipófise, pois ambas são originadas tanto do tecido neural quanto de tecido epitelial. A porção externa da glândula adrenal, conhecida com córtex, desenvolve- se de células mesodérmicas, na região ao redor do polo superior dos rins em desenvolvimento. Estas células formam cordões de células endócrinas epiteliais que dão origem as células esteroidogênicas. Logo depois da formação do córtex adrenal, as células originárias da crista neural associadas aos gânglios simpáticos, denominadas células cromafins, migram para dentro do córtex e são envoltas pelas células corticais. Assim, as células cromafins ocupam a porção interna da glândula adrenal, que é denominada medula (KOEPPEN; STANTON, 2009).

As glândulas adrenais compreendem duas regiões distintas: uma parte interna, ou medula, que secreta adrenalina (epinefrina) e noradrenalina (norepinefrina) e uma camada externa ou córtex, que é subdividida em três camadas: zona glomerulosa que secreta o mineralocorticoide aldosterona, zona fasciculada que produz, principalmente, o glicocorticoide cortisol e zona reticular produtora de hormônios androgênicos (BEAR; CONNORS; PARADISO, 2017; OLIVEIRA, 2013).

A zona fasciculada do córtex adrenal é a camada do meio e a mais larga, ocupando cerca de $75 \%$ do córtex adrenal e é responsável por secretar os glicocorticoides cortisol e corticosterona, bem como pequena quantidade de androgênios e estrogênios adrenais. A secreção dessa região é regulada, especialmente, pelo eixo hipotálamo-hipófise-adrenal (HHA) por meio do hormônio adrenocorticotrópico (ACTH) (GUYTON; HALL, 2011).

As percepções de dor causadas pelo estresse físico ou lesões nos tecidos são, inicialmente, transmitidas centralmente por meio do tronco cerebral e finalmente, para a eminência mediana do hipotálamo, onde o CRH é liberado para o sistema porta hipofisário. Rapidamente, toda a sequência de controle provoca a liberação de grande quantidade de cortisol no sangue. O estresse psicológico pode ocasionar elevação igualmente rápida da secreção de ACTH. Aceita-se que isso resulte do aumento no funcionamento do sistema límbico, particularmente na região da amígdala e do hipocampo, que transmitem, então, estímulos para o hipotálamo. A figura 2 mostra que o próprio cortisol atua negativamente (feedback negativo) sobre o hipotálamo e a pituitária para manter o seu ponto de equilíbrio. Contudo, estudos demonstraram que os estímulos estressantes são preponderantes. Desta forma, o estresse pode se impor sobre o feedback inibitório direto do cortisol, provocando exacerbações na secreção do cortisol (GUYTON; HALL, 2011).

Situações de estresse crônico estão quase sempre associados a efeitos deletérios importantes relacionados a condições clínicas crônicas e degenerativas, tais como aumento da resistência insulínica, aterosclerose, maior deposição de gordura visceral, osteoporose, alterações imunológicas e transtornos psiquiátricos. (BORGES NETO, 2011; MATOS, 2011). Neste sentido, o presente estudo, baseado na revisão de literatura, teve por objetivo elucidar quais são as principais patologias físicas e mentais que podem acometer os indivíduos que estão submetidos ao estresse crônico.

\section{2- DISCUSSÃO}

\section{1 - O Estresse e as Patologias Físicas}

2.1.1- O Estresse e o Encéfalo

O estresse leva à liberação do hormônio esteróide cortisol pelo córtex da adrenal. Dadas as suas características lipossolúveis, os glicocorticoides podem facilmente atravessar a barreira hematoencefálica e acessar o cérebro onde eles se ligam aos receptores. As três áreas cerebrais mais importantes que contêm receptores de glicocorticoides são o hipocampo, a amígdala e os lobos frontais, que são estruturas cerebrais conhecidas por estarem envolvidas nos processos de aprendizagem e memória (LUPIEN et al., 2007).

O cortisol, por meio da pela corrente sanguínea chega até o encéfalo e liga-se aos receptores no citoplasma de muitos neurônios. A ativação dos receptores desencadeia respostas no núcleo da célula, onde estimulam a transcrição gênica e, por consequência, a síntese proteica. Uma das ações do cortisol é a facilitação da entrada de mais $\mathrm{Ca} 2+$ nos neurônios através de canais iônicos dependentes de voltagem. Isso pode ocorrer devido a uma alteração direta nos canais, ou pode ser causado indiretamente, por mudanças no metabolismo energético celular. Independente da causa, o maior influxo de $\mathrm{Ca} 2+$ nos neurônios aumenta a atividade de enzimas intracelulares que degradam ácidos nucleicos, proteínas e lipídeos. Os neurônios literalmente se autodigerem (BEAR; CONNORS; PARADISO, 2017).

Por questões éticas, orgânicas e de biossegurança, estudar o encéfalo humano é tarefa extremamente difícil. Por isso, pesquisadores vêm estudando encéfalos de pequenos animais, como por exemplo, encéfalos de ratos. Diversos estudos 
revelaram que administração diária de corticosterona (o glicocorticoide do rato, análogo ao cortisol humano) por várias semanas levaram à redução dos dendritos em muitos neurônios com receptores para corticosterona. Poucas semanas mais tarde, essas células começaram a morrer. Resultados semelhantes foram achados quando, em vez de receberem injeções diárias de hormônios, os ratos foram estressados durante inúmeros dias consecutivos (BEAR; CONNORS; PARADISO, 2017).

Pesquisas têm mostrado claramente que o estresse crônico causa envelhecimento prematuro do encéfalo. Em humanos, exposição aos horrores da guerra, abuso sexual e outros tipos de violência extrema podem levar ao transtorno do estresse pós-traumático, com sintomas de ansiedade, distúrbios de memória e pensamentos recorrentes. As pesquisas com imagens têm consistentemente encontrado alterações degenerativas nos encéfalos das vítimas, especialmente no hipocampo (BEAR; CONNORS; PARADISO, 2017).

Atualmente, inúmeras pesquisas têm buscado compreender a resposta cognitiva das crianças submetidas ao estresse. $\mathrm{O}$ estresse e seus hormônios afetam negativamente a recuperação das informações armazenadas por crianças. Para comprovar tal afirmação, um estudo alemão com 44 crianças a termo foram aleatoriamente encaminhadas a uma situação estressante ou não estressante (situação controle), após, aprenderem a localização de 15 pares de figuras em um jogo de memória. O cortisol e a alfa-amilase foram mensurados imediatamente antes e mais três vezes após a exposição ao agente estressor ou situação controle (+01 min, $+10 \mathrm{~min},+25 \mathrm{~min})$. Comparadas ao grupo controle, as crianças que foram submetidas ao estresse tiveram um considerável aumento de cortisol e mais dificuldades em relembrar a posição das figuras. Além disso, observou-se, dentre as crianças estressadas, uma positiva correlação entre aumento de cortisol e número de erros cometidos durante a evocação do posicionamento dos pares de figuras no jogo de memória (QUESADA et al., 2012).

Quesada (2013) avaliou os efeitos da prematuridade no funcionamento do eixo HHA e do sistema nervoso simpático na memória, no desenvolvimento comportamental e emocional em crianças. Foram constatadas alterações no funcionamento do eixo HHA em crianças pré- termo, principalmente em meninas, quando comparadas a crianças a termo. $\mathrm{O}$ aumento de cortisol em resposta ao estressor foi maior em meninas pré-termo do que em meninos pré- termo. Além disso, crianças pré- termo apresentaram mais sintomas emocionais, déficits de memória visual e dificuldades em recuperar as informações verbais armazenadas quando comparadas as a termo. $\mathrm{O}$ estudo sugeriu que a exposição a inúmeros procedimentos invasivos e dolorosos durante os cuidados neonatais contribuíram para estas alterações (QUESADA et al., 2012).

\section{2- Elevação da Concentração Sanguínea de Glicose}

$\mathrm{O}$ efeito metabólico mais bem estudado do hormônio cortisol e de outros glicocorticoides é sua capacidade de estimular a gliconeogênese (formação de carboidratos a partir de aminoácidos, lactato e glicerol) pelo fígado, cuja atividade frequentemente aumenta por até 10 vezes. Isso ocorre porque o cortisol estimula a síntese das enzimas necessárias para a conversão de aminoácidos em glicose pelas células hepáticas. A maior produção de enzimas resulta do efeito dos glicocorticoides sobre a ativação da transcrição de DNA nos núcleos das células hepáticas, com a formação de RNAs mensageiros que, por sua vez, sintetizam as enzimas necessárias para a gliconeogênese (GUYTON; HALL, 2011).

Além disso, os aminoácidos dos tecidos extrahepáticos, principalmente, do tecido muscular são mobilizados pelo cortisol. Como resultado, mais aminoácidos são enviados para o plasma sanguíneo para entrar no processo de gliconeogênese no fígado e, portanto, promover a formação de glicose (GUYTON; HALL, 2011).

O cortisol também é responsável pela redução moderada da utilização de glicose na maior parte das células do organismo. Apesar da causa dessa redução não ser totalmente conhecida, a maioria dos pesquisadores acreditam que o cortisol retarde diretamente a velocidade de utilização de glicose, em algum local entre sua entrada nas células e sua degradação final (GUYTON; HALL, 2011).

Tanto a redução da taxa de utilização da glicose pelos tecidos corporais quanto o aumento da gliconeogênese provocam a elevação da concentração sanguínea de glicose. Por sua vez, a elevação da concentração da glicose estimula a secreção de insulina. Os maiores níveis plasmáticos de insulina, entretanto, não são tão eficazes na manutenção de níveis normais da glicose plasmática. Por motivos não inteiramente elucidados, os altos níveis de glicocorticoides reduzem a sensibilidade tecidual, especialmente do músculo esquelético e tecido adiposo, ao efeito hipoglicemiante da insulina (GUYTON; HALL, 2011). 
O aumento da concentração sanguínea de glicose é, em alguns casos, tão alto (50\% ou mais, acima do normal) que a patologia é conhecida como diabetes adrenal. A utilização de insulina reduz moderadamente a concentração sanguínea de glicose no diabetes adrenal porque os tecidos se tornam resistentes aos efeitos da insulina (GUYTON; HALL, 2011; PENTEADO; OLIVEIRA, 2009).

Andrade e Alves (2018) estudaram a influência dos fatores socioeconômicos e psicológicos no controle glicêmico de crianças pequenas portadoras de diabetes mellitus tipo 1. Eles observaram uma associação negativa entre o controle glicêmico (níveis de hemoglobina glicada), status socioeconômico e condição psicológica. Ou seja, as crianças submetidas aos estresses socioeconômicos e psicológicos apresentaram mais chances de apresentar valores indesejados de hemoglobina glicada (ANDRADE; ALVES, 2018).

\section{3- Redução das Proteínas Celulares}

Um dos efeitos marcantes do cortisol nos sistemas metabólicos do organismo é a depleção das reservas proteicas em, praticamente, todas as células corporais, exceto no fígado. Este quadro é originado pela diminuição na produção de proteínas, além do maior catabolismo das proteínas pré-existentes nas células. Ambos os efeitos podem resultar parcialmente na redução do transporte de aminoácidos para os tecidos extra-hepáticos e, principalmente, porque o cortisol também reduz os processos de transcrição e a subsequente síntese proteica em muitos tecidos extrahepáticos, especialmente nos músculos e nos tecidos linfoides (GUYTON; HALL, 2011).

\section{4- Fraqueza Muscular}

$\mathrm{Na}$ presença de altas taxas de produção de cortisol, as fibras musculares podem ficar tão fracas que o indivíduo pode apresentar dificuldades para se levantar da posição agachada. Isso ocorre devido à redução da síntese proteica e do aumento do catabolismo das proteínas musculares que terão seus aminoácidos desviados para o processo de gliconeogênese (AMOEDO, 2010; GUYTON; HALL, 2011).

\subsection{1- Estrias}

Até mesmo as proteínas das fibras colágenas no tecido subcutâneo são parcialmente metabolizadas, de modo que os tecidos subcutâneos se tornam frágeis, resultando em grandes estrias arroxeadas em várias partes do corpo, em especial no abdome (GUYTON; HALL, 2011).

\subsection{2- Osteoporose}

Exacerbações no funcionamento da glândula adrenal com liberação de altas taxas de cortisol provocam redução significativa da deposição proteica por todo o organismo, aumento do catabolismo proteico, além de especificamente diminuir a atividade osteoblástica (GUYTON; HALL, 2011).

Todos os ossos do corpo estão em constante remodelação, sendo os osteoclastos responsáveis pela reabsorção (retirada) óssea, enquanto os osteoblastos são os formadores do novo osso que preencherá as lacunas deixadas pelos osteoclastos. Quando o glicocorticoide deprime a atividade osteoblástica, o osso passa a ser mais reabsorvido do que depositado e assim, passam a ficar enfraquecidos (GUYTON; HALL, 2011; LANNA; MONTENEGRO JR.; PAULA, 2003).

\section{5- Obesidade Causada por Excesso de Cortisol}

As altas taxas de cortisol oriundas do estresse crônico podem desregular o eixo HHA e promover um aumento na ingestão de alimentos de alta palatabilidade e a deposição de gordura (essencialmente visceral), devido à interação com vários componentes endócrinos. Além disso, os glicocorticoides podem diminuir a produção de hormônios tireoidianos e assim, diminuir a taxa de metabolismo basal de forma a favorecer o ganho de peso. Os elevados níveis de estresse podem ativar centros cerebrais de motivação e recompensa, de modo que o organismo passa a desejar alimentos de maior palatabilidade, geralmente, mais calóricos (MELIN; PINHÃO; CORREIA, 2013).

Inúmeras pessoas com excesso de secreção de cortisol desenvolvem tipo peculiar de obesidade, com deposição excessiva de gordura no tórax e na cabeça, gerando sinais clínicos chamados de "giba de búfalo" e "face em lua cheia". Embora sua causa seja desconhecida, foi sugerido que essa obesidade resulte do estímulo excessivo a ingestão alimentar, de modo que a gordura seja gerada em alguns tecidos mais rapidamente do que é mobilizada e oxidada (GUYTON; HALL, 2011).

\section{6- Efeito do Cortisol sobre a Imunidade}

O cortisol em excesso tem a capacidade de reduzir o número de eosinófilos e linfócitos sanguíneos. Esse efeito começa alguns minutos após a administração de cortisol e fica acentuado após algumas horas (GUYTON; HALL, 2011).

Sendo assim, a linfocitopenia ou eosinopenia constituem um critério diagnóstico importante para a superprodução de cortisol pelas adrenais. Além do mais, a utilização de altas doses de cortisol ocasiona atrofia 
significativa de todos os tecidos linfoides do organismo, o que reduz a produção de células $\mathrm{T}$ e de anticorpos. Com isso, o nível da imunidade contra inúmeros patógenos é reduzido. Isso pode, ocasionalmente, causar infecções graves e morte por doenças, que em condições normais não seriam letais, tais como a tuberculose em pessoa cuja doença havia sido controlada antes (GUYTON; HALL, 2011; MATTOS, 2011).

Contudo, a capacidade do cortisol e de outros glicocorticoides de diminuir a imunidade os torna ferramentas úteis na prevenção da rejeição imunológica de corações, rins e outros órgãos transplantados (GUYTON; HALL, 2011; MATTOS, 2011).

\section{7- Hipertensão Arterial}

O cortisol possui baixa ação mineralocorticoide, porém, é produzido em grande quantidade. Os mineralocorticoides receberam esse nome por atuarem, especificamente, nos eletrólitos ("minerais") dos líquidos extracelulares, em especial sódio e potássio (AMOEDO, 2010; GUYTON; HALL, 2011).

Em condições orgânicas normais, a função mineralocorticoide do cortisol é pouco expressiva, apenas recebendo papel importante nas situações de aumento anormal de liberação do cortisol. A aldosterona e o cortisol (em menor escala) promovem a maior reabsorção do sódio pelos túbulos renais (AMOEDO, 2010; GUYTON; HALL, 2011).

Quando ocorre excesso de sal no líquido extracelular, a osmolaridade do líquido aumenta, o que estimula a maior reabsorção de água para normalizar a concentração extracelular de sal. Isso aumenta o volume do líquido extracelular. Além disso, o aumento da osmolaridade causado pelo sal em excesso no líquido extracelular estimula também o mecanismo secretor do hipotálamo-hipófise posterior, que passa a secretar maior quantidade de hormônio antidiurético (ADH), também conhecido como vasopressina. Esse hormônio então faz com que os rins reabsorvam quantidade maior de água pelos túbulos renais, reduzindo o volume de urina e elevando o volume do líquido extracelular (AMOEDO, 2010; GUYTON; HALL, 2011).

O aumento do volume do líquido extracelular aumenta o volume sanguíneo, consequentemente, aumentando a pressão arterial (GUYTON; HALL, 2011).

\section{3- CONSIDERAÇÕES FINAIS}

As reações fisiológicas ao estresse são essenciais para a sobrevivência e adaptação das espécies, pois liberam energia (aumento da concentração de glicose plasmática), aumentam os batimentos cardíacos e o fluxo sanguíneo para os músculos, ajustando o corpo para lidar com os estressores, em reações de luta ou fuga. Além disso, o cortisol possui vários benefícios como, por exemplo, a resolução dos processos inflamatórios dentro de horas ou de alguns dias, com aumento da regeneração tecidual. Ainda, o cortisol e outros glicocorticoides possuem a capacidade de reduzir substancialmente a imunidade, os tornando agentes uteis para a prevenção da rejeição imunológica de órgãos transplantados (GUYTON; HALL, 2011).

Contudo, exposição a altos níveis de cortisol devido à prolongada estimulação do eixo HHA e do sistema nervoso autônomo podem representar um risco à saúde dos indivíduos. Para ilustrar o modo como concentrações elevadas de cortisol podem prejudicar a saúde humana, basta entender a doença de Cushing. Nessa doença, o surgimento de tumores na hipófise ocasiona à hipersecreção crônica de ACTH e, consequentemente, ao hipercortisolismo. Os portadores dessa doença são mais suscetíveis a doenças crônicas, problemas cardíacos, diabetes e déficits de memória (GUYTON; HALL, 2011).

Ainda, é importante destacar que o estresse atua negativamente nas funções fisiológicas de adultos e crianças, sendo os quadros agravados quando os indivíduos são expostos a traumas como, por exemplo, violência doméstica ou abuso sexual.

Ademais, o estresse pode provocar um mau funcionamento do eixo hipotálamo- hipófise-tireoide, pois, o CRH apresenta um importante papel na inibição da secreção de hormônios, dentre os quais o hormônio liberador de tireotropina (TRH) e o hormônio tireoestimulante (TSH), diminuindo as funções da tireoide (TSIGOS; CHROUSOS, 2002; VILELA, 2014). O hormônio TSH atua diretamente na tireoide para estimular a produção dos hormônios triiodotironina (T3) e tiroxina (T4).

É preciso destacar que as pessoas com estresse crônico não necessariamente apresentarão todos os sinais e sintomas oriundos do funcionamento anormal do eixo HHA. Afinal, como apresentado, o estresse possui um caráter individual, sendo a resposta diferente entre os indivíduos. Ou seja, os mesmos poderão apresentar uma ou mais patologias, sendo o grau de adoecimento resultado de fatores genéticos, cognitivos, estilo de vida e experiências anteriores.

Para minimizar o estresse imposto as pessoas cotidianamente é necessário adotar medidas que podem ser divididas em dois grupos: as de cunho pessoal e as de responsabilidade do Estado. Nas 
medidas pessoais de combate ao estresse destacamse a necessidade das pessoas se preocuparem menos com as coisas que não podem ser mudadas, buscar realizar atividades que são prazerosas, se cobrar menos e comemorar mais, desenvolver a inteligência emocional e eliminar hábitos estressantes. O acompanhamento por um profissional devidamente habilitado pode contribuir para o alcance de resultados satisfatórios.

Dentre as obrigações do Estado em promover saúde à população é importante que o mesmo ofereça condições dignas de trabalho, acesso à saúde de qualidade, saneamento básico, mobilidade urbana, segurança pública, segurança alimentar, políticas habitacionais e opções de lazer, cultura e esporte.

Em suma, toda e qualquer ação que ofereça paz, tranquilidade e estabilidade as pessoas pode evitar o estresse e, por consequência, minimizar as suas patologias associadas.

Neste sentido, Oliveira (2013) avaliou se a modalidade terapêutica de imposição de mãos - Reiki - seria capaz de produz alterações de parâmetros psicofisiológicos e de qualidade de vida, em voluntários idosos com sintomas de estresse. $\mathrm{O}$ autor demonstrou que houve a redução dos níveis de estresse, dos níveis de ansiedade e depressão e redução da percepção de tensão muscular, além, da elevação do bem estar.

\section{REFERÊNCIAS BIBLIOGRÁFICAS}

AMOEDO, C. (Coord.). Hipertensão arterial sistêmica secundária. In: NOBRE, F. (Ed.). VI Diretrizes Brasileiras de Hipertensão - DBH VI. Ribeirão Preto: Revista Brasileira de Hipertensão, 2010. p. 44 - 51.

ANDRADE, C. J.; ALVES, C.A. Influence of socioeconomic and psychological factors in glycemic control in young children with type 1 diabetes mellitus. Jornal de Pediatria, Rio de Janeiro, 2018. No prelo.

BEAR, M. F.; CONNORS, B. W.; PARADISO, M. A. Neurociência: desvendando o sistema nervoso. 4. ed. Porto Alegre: Artmed, 2017. ISBN: 9788582714324.

BORGES NETO, J. B. Os neurotransmissores. In: FIKS, J. P.; MELLO, M. F. Transtorno do estresse pós-traumático: violência, medo e trauma no Brasil. São Paulo: Atheneu, 2011. p. 49-64.

GUYTON, A. C.; HALL, J. E. Tratado de fisiologia médica. 10. ed. Rio de Janeiro: Guanabara Koogan, 2011. ISBN 978-85-352-37351.

KOEPPEN, B. M.; STANTON, B. A. (Eds.) Fisiologia. 6. ed. Rio de Janeiro: Elservier, 2009. ISBN 978-85-352-3057-4.

LANNA, C. M. M.; MONTENEGRO JR., R. M.; PAULA, F. J. A. Fisiopatologia da

osteoporose induzida por glicocorticoide. Arquivos Brasileiros de Endocrinologia e Metabologia, Ribeirão Preto, v. 47, n. 1, p. $9-18$, fev. 2003.

LIPP, M. E. N. Mecanismos neuropsicofisiológicos do stress: teoria e aplicações clínicas.

São Paulo: Casa do Psicólogo, 2010, 227 p.

LUPIEN, S. J.; MAHEU, F.; TU, M.; FIOCCO, A.; SCHRAMEK, T. E. The effects of stress

and stress hormones on human cognition: Implications for the field of brain and cognition.

Brain and Cognition, Vancouver, v. 65, n. 3, p. 209-237, dez. 2007.

MATTOS, P. A psiconeuroendocrinologia. In: FIKS, J. P.; MELLO, M. F. Transtorno do estresse pós-traumático: violência, medo e trauma no Brasil. São Paulo: Atheneu, 2011, p. 77-89.

MELIN, D.; PINHÃO, S.; CORREIA, F. Stresse e sua relação com a obesidade. Alimentação Humana, Porto, v. 19, n. 3, p. 74-81, 2013. OLIVEIRA, RICARDO MONEZI JULIÃO de. Efeitos da prática do Reiki sobre aspectos psicofisiológicos e de qualidade de vida de idosos com sintomas de estresse: estudo placebo e randomizado. 165 f. Tese (Doutorado em Ciências) - Escola Paulista de Medicina, Universidade Federal de São Paulo, São Paulo, 2013.

PENTEADO, M. S.; OLIVEIRA, T. C. Associação estresse-diabetes mellitus tipo II. Revista da Sociedade Brasileira de Clínica Médica, Botucatu, v. 7, n. 1, p. 40-45, jan./fev. 2009.

QUESADA, ANDREA AMARO. Estresse e prematuridade: um risco para a memória e para o desenvolvimento emocional na infância. 67 f. Tese (Doutorado em Ciências Médicas)

- Faculdade de Medicina, Universidade de Brasília, Brasília, 2013.

QUESADA, A. A.; WIEMERS, U. S.; SCHOOFS, D.; WOLF, O. T. Psychosocial stress

exposure impairs memory retrieval in children. Psychoneuroendocrinology, v. 37, n. 1, p. 125-136, 2012.

SILVA, J. J. Estresse: o impulso da vida. São Caetano do Sul (SP): Yendis Editora, 2005. ISBN 8598859176.

TSIGOS, C.; CHROUSOS, G. P. Hypothalamic-pituitary-adrenal axis, neuroendocrine factors and stress. Journal of psychosomatic research, v. 53, n. 4, p. 865-871, out. 2002.

VILELA, LUCIA HELENA MORAES. Relação da depressão com os eixos hipotálamo- hipófise-adrenal, hipotálamohipófise-tireoide e o estresse precoce. 167 p. Tese (Doutorado em Ciências Médicas) - Faculdade de Medicina de Ribeirão Preto, Ribeirão Preto, 2014. 